\title{
Troxerutin Preconditioning Modulates the Activity of TLR4/NF-kB Signaling Pathway and Mitochondrial ATP-Sensitive Potassium Channels in a Rat Model of Myocardial Ischemia/Reperfusion Injury
}

\section{Farid Masoud}

Tabriz University of Medical Sciences Drug Applied Research Center

\section{Behnaz Mokhtari}

Tabriz University of Medical Sciences Drug Applied Research Center

\section{Sanaz Gholami}

Tabriz University of Medical Sciences Faculty of Medicine

Aniseh Javadi

Tabriz University of Medical Sciences Faculty of Medicine

Reza Badalzadeh ( $\square$ badalzadehr@tbzmed.ac.ir)

Tabriz University of Medical Sciences https://orcid.org/0000-0002-8092-7820

\section{Research Article}

Keywords: Myocardial ischemia/reperfusion injury, inflammation, TLR4/NF-kB pathway, mitochondrial KATP channel, troxerutin.

Posted Date: May 20th, 2021

DOl: https://doi.org/10.21203/rs.3.rs-508614/v1

License: (c) (i) This work is licensed under a Creative Commons Attribution 4.0 International License.

Read Full License 


\section{Abstract \\ Purpose}

Given the rising prevalence of ischemic heart diseases (IHD), investigations for achieving effective treatments are required. The purpose of this study is further understanding of troxerutin's impact on inflammatory response induced by myocardial ischemia/reperfusion (MI/R) injury, and the role of toll-like receptor 4 (TLR4)-nuclear factor kappa B (NF-KB) signaling pathway and mitochondrial ATP-sensitive potassium ( mitoK $\left._{\text {ATP }}\right)$ channels in this scenario.

\section{Methods}

Sixty male Wistar rats were randomly divided into six groups: Control, troxerutin-receiving, MI/R, MI/R plus troxerutin, MI/R plus 5-hydroxydecanoate (5-HD), and MI/R plus troxerutin and 5-HD. The Langendorff-perfused hearts of animals were subjected to $30 \mathrm{~min}$ of left anterior descending coronary artery (LAD) ligation and $45 \mathrm{~min}$ of reperfusion. Troxerutin $(150 \mathrm{mg} / \mathrm{kg} /$ day) was administered for 4 weeks prior to MI/R. 5-HD $(100 \mu \mathrm{M})$ was added to the perfusion solution $20 \mathrm{~min}$ before the ischemia. Myocardial infarct size (IS), lactate dehydrogenase (LDH) release, the expressions of TLR4 and NF-KB, and the levels of tumor necrosis factor-alpha (TNF- $\alpha$ ) and interleukin-1beta (IL-1 $\beta$ ) were measured.

\section{Results}

Troxerutin preconditioning significantly reduced IS $(P<0.05)$ and LDH release $(P<0.05)$. Pretreatment with troxerutin decreased the expressions of TLR4 $(P<0.05)$ and NF-KB $(P<0.01)$, and reduced the levels of TNF- $\alpha(P<0.01)$ and IL-1 $\beta(P<0.05)$. Inhibition of mitoK ATP $_{\text {chen }}$ channels by $5-H D$ significantly reversed the cardioprotective effects of troxerutin $(P<0.05)$.

\section{Conclusion}

The present work revealed that troxerutin preconditioning has cardioprotective effects against $\mathrm{MI} / \mathrm{R}$ damage, which are partly mediated through anti-inflammatory effects and the activation of mitoK $\mathrm{K}_{\text {ATP }}$ channels.

\section{Introduction}

Cardiovascular diseases are the major cause of death worldwide, especially in industrialized societies. So protecting the heart against stressors and ischemic conditions is very importan [1]. Myocardial ischemia/reperfusion (MI/R) injury is the tissue injury resulted when blood supply restores to the tissue following ischemia. Despite the fact that early reperfusion of the myocardial ischemic region is essential 
for the survival of cardiomyocytes, but reperfusion itself leads to the destructive responses in circulating cells and cardiomyocytes, and a series of pathophysiological and biochemical changes referred as reperfusion injury $[2,3]$. It has been shown that $\mathrm{MI} / \mathrm{R}$ injury leads to the contractile dysfunction, arrhythmias and increased infarct size by increasing cardiomyocytes apoptosis and necrosis [4].

The pathophysiology of MI/R injury is complex, and several mechanisms mediate the detrimental effects of $I / R$ injury [5-8]. One of the main mechanisms that causes MI/R injury is the activation of inflammatory pathways [9]. The inflammatory immune response has been shown to begin with ischemia and continue for several hours throughout the reperfusion phase. Toll-like receptor 4 (TLR4) is involved in the detrimental inflammatory effects under MI/R injury and participates in the formation of active oxygen free radicals, as well as activation of many cytokines $[4,10]$. TLR4 can activate and transfer the nuclear factor-kappa B (NF-KB) to the nucleus, thereby activating the transcription and releasing of inflammatory cytokines. Activation of NF-KB leads to the promotion of inflammatory genes expression such as tumor necrosis factor-alpha (TNF- $\alpha$ ) and interleukin-1 beta (IL-1 3 ). Accumulation of TNF- $a$ and IL-1 $\beta$ in the ischemic region causes tissue injury, oxygen free radicals releasing, cardiomyocyte damage and cardiac dysfunction $[10,11]$. Inflammation has recently been considered as a therapeutic target in myocardial I/R injury, but the results are controversial. Despite numerous studies, the application of strategies targeting the inflammatory cascades in patients with myocardial infarction has been failed [12]. Therefore, researches in this field and finding better strategies to combat with MI/R injury via the interventions in inflammatory pathways are of particular importance. Additionally, numerous studies have shown that the opening of mitochondrial ATP-sensitive potassium ( mitoK $_{\text {ATP }}$ ) channel in the outer membrane of the mitochondrion may play essential role in preventing cardiac I/R injury via maintaining mitochondrion integrity $[13,14]$. Opening of mitoK $\mathrm{ATP}_{\text {ATP }}$ channels has been shown to play a key role in the anti-apoptotic processes and cardioprotective mechanisms of ischemic preconditioning and postconditioning [15]. Therefore, investigations for finding effective strategies to target mitochondrial $\mathrm{K}_{\text {ATP }}$ channels in order to alleviate $\mathrm{MI} / \mathrm{R}$ injury are very necessary.

Troxerutin, also known as vitamin P4, is a natural bioflavonoid widely exists in tea, coffee, cereal sprouts, and various fruits and vegetables. It is derived from Sophora Japonica and Dimorphandra Gardneriana, and has anti-oxidant, anti-inflammatory, and anti-thrombotic activities [16-18]. Troxerutin has been commonly used in the treatment of chronic venous insufficiency [19]. It has been shown that troxerutin can reduce renal damage induced by d-galactose in mice by increasing the anti-oxidant activity and $\mathrm{H}_{2} \mathrm{O}_{2}$ cleavage, and reducing MDA production, as well as inhibition of NF-kB-mediated inflammatory response [16]. Also, troxerutin can attenuate reactive oxygen species (ROS) production and DNA oxidative injury in d- galactose-induced kidney injury in mice [20].

To our knowledge, a less promising study is existed to determine the cardioprotective activities of troxerutin against I/R injury, especially via modulation of the activity of TLR4/NF-KB signaling pathway and mitochondrial $\mathrm{K}_{\mathrm{ATP}}$ channel. Given the potential anti-inflammatory effect of troxerutin and the lack of a study in this area, we hypothesized that troxerutin might decrease cardiac l/R injury via affecting the 
TLR4/NF-KB signaling pathway and mitochondrial $\mathrm{K}_{\text {ATP }}$ channels. For that reason, the aim of the present study is to investigate the effect of troxerutin on myocardial infarct size, lactate dehydrogenase (LDH) release, TLR4 and NF-KB expressions, as well as TNF- $\alpha$ and IL- $1 \beta$ levels, and the role of mitoK ATP channels under $\mathrm{MI} / \mathrm{R}$ injury. Insights into these aspects are expected to contribute to a better understanding of troxerutin's effects on Ml/R injury.

\section{Materials And Methods}

\subsection{Animals and chemicals}

Healthy male Wistar rats at 3-4 months of age $(n=60,250-300 \mathrm{~g})$ were purchased from the animal center of Tabriz University of Medical Sciences. All the rats were housed in the animal room under a $12 \mathrm{~h}$ light/ $12 \mathrm{~h}$ dark schedule at a temperature of $25 \pm 2^{\circ} \mathrm{C}$ and a humidity of $50 \pm 10 \%$, and had free access to standard food and water. Study protocols and procedures were performed in accordance with the Guide for the Care and Use of Laboratory Animals published by the US National Institutes of Health (8th Edition, NRC 2011) and were approved by the ethical committee of Tabriz University of Medical Sciences (Ethical code: IR.TBZMED.VCR.REC.92-3729). Troxerutin was obtained from Sigma (St. Louis, MO, USA). 5-

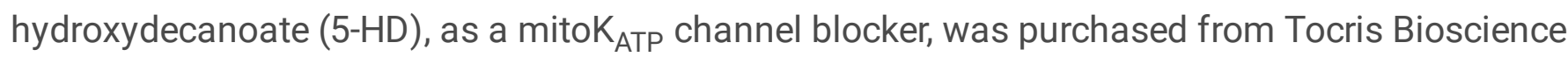
(Avonmouth, UK), all substances for the Krebs-Henseleit (K-H) solution were obtained from Merck Company (Munchen, Germany), and all other chemicals and reagents were obtained from commercial sources in the highest quality available.

\subsection{Langendorff isolated heart perfusion and induction of regional I/R protocol}

Animals were heparinized $(500 \mathrm{lU} / \mathrm{kg}$ ), then anesthetized with intraperitoneal injection of a combination of ketamine $(60 \mathrm{mg} / \mathrm{kg})$ and xylazine $(5 \mathrm{mg} / \mathrm{kg})$. After opening the chest in layers, the heart was rapidly removed and then mounted on a pressure-constant Langendorff perfusion apparatus (ML176-V; AD Instruments, New South Wales, Australia). The isolated heart was retrogradely perfused via the aorta with $\mathrm{K}-\mathrm{H}$ solution that contained (in mmol/L) $\mathrm{MgSO}_{4} 1.2 ; \mathrm{KCl} 4.8 ; \mathrm{NaCl} 118.5 ; \mathrm{NaHCO}_{3} 25 ; \mathrm{KH}_{2} \mathrm{PO}_{4} 1.2 ; \mathrm{CaCl}_{2}$

1.7 and glucose 11.1 at $\mathrm{pH} 7.4$ under a constant perfusion pressure of $80 \mathrm{~mm} \mathrm{Hg}$. The perfusion solution was gassed with a mixture of $95 \% \mathrm{O}_{2}$ and $5 \% \mathrm{CO}_{2}$ at $37 \pm 0.5^{\circ} \mathrm{C}$. Throughout the stabilization, the coronary flow rate was $12-14 \mathrm{ml} / \mathrm{min}$. For maintaining the temperature near $37^{\circ} \mathrm{C}$, the isolated heart was surrounded by a homoeothermic glass cover. Before the induction of regional ischemia, the isolated hearts in Langendorff setting were perfused with $\mathrm{K}-\mathrm{H}$ solution for 15-20 minutes to stabilize cardiac activity. Then regional ischemia was done by ligation of the left anterior descending (LAD) coronary for $30 \mathrm{~min}$, close to its origin. Reperfusion was done by reopening of the LAD for $45 \mathrm{~min}$. The efficiency of coronary ligation and sufficient perfusion were approved by coronary flow. Declining at least $25 \%$ in coronary flow during occlusion was considered satisfactory.

\subsection{Experimental design}


Rats were randomly divided into the following six experimental groups ( $\mathrm{n}=10$ in each group):

(1) Control: non-treated healthy group without experiencing I/R; (2) TXR: troxerutin-receiving group without experiencing I/R; (3) MI/R: non-treated group experiencing I/R; (4) MI/R + TXR: troxerutin-receiving group experiencing I/R; (5) Ml/R + 5-HD: 5-HD-receiving group experiencing I/R; and (6) Ml/R + TXR + 5$\mathrm{HD}$ : troxerutin plus $5-\mathrm{HD}$-receiving group experiencing $\mathrm{l} / \mathrm{R}$.

In TXR-receiving groups, troxerutin $(150 \mathrm{mg} / \mathrm{kg}$ ) was orally administrated through a gavage tube once a day for 4 weeks before the induction of MI/R. In 5-HD-receiving groups, the hearts were perfused with a K-

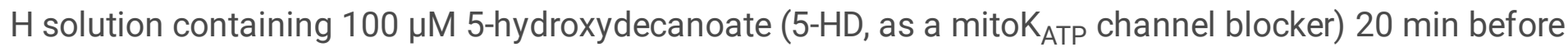
the ischemia. After $45 \mathrm{~min}$ of reperfusion, biochemical evaluations were performed from tissue sampling $(n=5)$, and the infarct size (IS) were evaluated $(n=5)$.

\subsection{Evaluation of area at risk and infarct size}

In order to differentiate the ischemic and non-ischemic areas from each other, the LAD was reoccluded following $45 \mathrm{~min}$ of reperfusion, then $0.25 \%$ Evans blue dye (3-4 ml, Sigma) was infused via the aortic cannula into the coronary system. After the hearts were frozen at $-20^{\circ} \mathrm{C}$ for $24 \mathrm{~h}$, the hearts were sliced into $2 \mathrm{~mm}$ sections, then incubated for $15 \mathrm{~min}$ in 1\% 2,3,5-triphenyltetrazolium chloride phosphatebuffered solution ( $\mathrm{pH} \mathrm{7.4)}$ at $37^{\circ} \mathrm{C}$. Hence, the slices were placed in $10 \%$ formalin for $24-48 \mathrm{~h}$. By means of Image $\mathrm{J}$ software ( $\mathrm{NIH}$, Bethesda, USA), the determination of infarct size was performed. Accordingly, the area at risk (AAR) was reported as a percentage of left ventricle (AAR/LV $\times 100)$, and IS was reported as a percentage of AAR (IS/AAR × 100).

\subsection{Measurement of lactate dehydrogenase (LDH) release}

$\mathrm{LDH}$ release into the coronary effluent was measured by a colorimetric method using the specific laboratory kit (Pars Azmoon Co., Karaj, Iran) and autoanalyzer (Alcyon 300, Abbott Labs, Santa Clara, CA, USA) in compliance with the manufacturer's methods. A spectrophotometer detected the absorbance of the solution for LDH at $492 \mathrm{~nm}$ [21]. The results are reported in IU/L.

\subsection{Preparation of tissue homogenates}

$45 \mathrm{~min}$ after reperfusion, the heart samples isolated from the ischemic zone of left ventricle were immediately frozen in liquid nitrogen and stored at $-80^{\circ} \mathrm{C}$. The preparation of samples was in accordance to a modified protocol for tissue homogenization. The ventricular tissue was weighed, then cut into pieces in about $5 \mathrm{ml}$ of ice-cold lysis buffer ( $1 \mathrm{mM}$ EDTA, $1 \mathrm{mM} \mathrm{KCL}, 1 \mathrm{mM} \mathrm{Na}_{3} \mathrm{VO}_{4}, 1 \mathrm{mM}$ $\mathrm{KH}_{2} \mathrm{PO}_{4}, 50 \mathrm{mM}$ Tris-HCl pH 7.4, $1 \mathrm{mM} \mathrm{NaF}, 1 \%$ triton X100 and protease inhibitor cocktail) and then homogenized by means of a Polytron PT-10/ST homogenizer. After centrifuging the homogenate $(10,000$ $R C F, 10 \mathrm{~min}, 4^{\circ} \mathrm{C}$ ), the supernatants were removed from the homogenates and rapidly frozen at $-80^{\circ} \mathrm{C}$. To determine protein concentration in samples, Bradford method was used.

\subsection{Western blotting}


TLR4 and NF-KB in isolated hearts of all groups were measured by western blotting. Fresh-frozen samples were dissected and homogenized in radio-immunoprecipitation assay (RIPA) lysis buffer (Sigma-Aldrich, St. Louis, MO, USA). The resulting solutions were centrifuged at 13,000 g for $20 \mathrm{~min}$. Next, the supernatants were collected. Using UV 3000 ultraviolet spectrophotometer (NanoDrop, Wilmington, $\mathrm{DE})$, the concentration of total protein was determined. Then, equal amounts of proteins $(50 \mu \mathrm{g})$ were loaded into the electrophoresis chamber in 10-15\% SDS-polyacrylamide gel. After that, separated soluble proteins were electro-transferred to a polyvinylidene-difluoride membrane (PVDF, Sigma-Aldrich, St. Louis, MO, USA), then incubated with $5 \%$ non-fat dry milk solution in Tris buffered saline-Tween 20 (TBST, pH 7.4) in room temperature for $2 \mathrm{~h}$ to block non-specific bindings. The membranes were incubated with primary antibodies at 1:1000 against TLR4 and NF-KB (Cell Signaling Technology, USA) diluted in blocking buffer overnight at $4^{\circ} \mathrm{C}$ on a shaker. To control for equal loading, membranes were also incubated for $\beta$-actin antibody (1:1000, Cell Signaling Technology, USA), diluted in TBST overnight at $4^{\circ} \mathrm{C}$. Then blots were incubated with goat anti-rabbit horseradish peroxidase conjugated Ig $\mathrm{G}$ for $1 \mathrm{~h}$ at room temperature. For detecting the immunoreactivity, protein bands were incubated with enhanced chemiluminescence (ECL) substrate (Millipore) and then were exposed to X-ray hyperfilm inside a hypercassette in a darkroom for $5 \mathrm{~min}$, and the chemiluminescence of the antibody binding was visualized using a visualizing machine. In order to quantify the intensity of protein bands in the blots, densitometry analysis was used and then normalized with the intensity of $\beta$-actin band as loading control. The measured values are reported in arbitrary unit.

\subsection{Determination of inflammatory cytokines}

For measuring the levels of inflammatory cytokines (TNF-a and IL-1 $\beta$ ), rat-specific enzyme-linked immunosorbent assay (ELISA) kits (eBioscience, Bender Medsystems, Austria) were used according to the manufacturer's methods. First, frozen samples get warmer in room temperature, then mixed gently. 50 $\mathrm{ml}$ samples and standards were added in duplicate to the 96-well plate. After adding the polyclonal antibodies separately to all wells, their surface were covered and incubated for $2 \mathrm{~h}$ at room temperature. Following washing, streptavidin-HRP was added to all of the wells and were incubated for $1 \mathrm{~h}$ at room temperature. TMB substrate solution was added to all of the wells. In order to hamper the enzyme reaction, the stop solution was added into the each well. Using a spectrophotometer, the relative absorbance of cytokines was read at $450 \mathrm{~nm}$. The resultant values were normalized and expressed as $\mathrm{pg} / \mathrm{mg}$ of protein in each sample. The limits of detection of rat TNF- $\mathrm{a}$ and IL-1 $\beta$ with ELISA kits were determined to be 11 and $4 \mathrm{pg} / \mathrm{ml}$ up to $2500 \mathrm{pg} / \mathrm{ml}$, respectively. The calculated overall intra-assay and inter-assay coefficients of variation for TNF-a were $<5$ and $<10 \%$ and for IL- $1 \beta$ were $<10$ and $<10 \%$, respectively. According to the manufacturer's assays, these ELISA kits show no cross-reactivity with the other cytokines and molecules.

\subsection{Statistical analysis}

The analysis was carried out using SPSS software 25 (SPSS Inc., Chicago, IL, USA). In order to determine the statistical significances between groups, one-way analysis of variance (ANOVA) followed by Tukey 
post hoc test was used. All values are expressed as means \pm standard errors of means (SEM). P value below $0.05(P<0.05)$ was considered as statistically significant difference.

\section{Results}

\subsection{Myocardial area at risk (AAR) and infarct size (IS)}

As shown in Fig. 1, there were no significant differences in the area at risk between all groups. The IS was significantly increased in MI/R group as compared to Control group $(P<0.001)$. Preadministration of troxerutin significantly reduced IS as compared to $\mathrm{MI} / \mathrm{R}$ hearts $(P<0.05)$. Adding $5-\mathrm{HD}$ to the perfusion solution significantly abolished the IS lowering effect of troxerutin $(P<0.05)$. However, there was no significant change in IS between MI/R and MI/R + 5-HD groups (Fig. 1).

\section{2. $\mathrm{LDH}$ release}

Figure 2 shows the levels of LDH release into the coronary effluent of treated and untreated I/R hearts. The levels of LDH release were significantly increased in MI/R group as compared to Control group $(\mathrm{P}<$ 0.001). Preadministration of troxerutin significantly reduced the levels of LDH release into the coronary effluent during reperfusion as compared to MI/R hearts $(P<0.05)$. Adding $5-H D$ to the perfusion solution significantly abolished the LDH lowering effect of troxerutin $(P<0.05)$. No significant differences were found in LDH release between Ml/R and Ml/R + 5-HD groups (Fig. 2).

\subsection{TLR4 expression}

As shown in Fig. 3, the expression of TLR4 in MI/R group was significantly increased as compared to Control group $(P<0.01)$. Preadministration of troxerutin significantly reduced the expression of TLR4 as compared to $M I / R$ hearts $(P<0.05)$. However, troxerutin in the presence of $5-H D$ could not significantly decrease the expression of TLR4 as compared to MI/R + TXR group. In other words, administration of 5$H D$ reversed the effect of troxerutin on the alteration of TLR4 expression. No significant differences were found in TLR4 expression between MI/R and Ml/R + 5-HD groups (Fig. 3).

\subsection{NF-kB expression}

Figure 4 shows the effect of troxerutin on the expression of NF-KB in treated and untreated I/R hearts. The expression of NF-KB in Ml/R group was significantly increased as compared to Control group $(P<0.001)$. Preconditioning with troxerutin significantly reduced the expression of NF-KB as compared to MI/R hearts $(P<0.01)$. Blocking the mitoK ATP $_{\text {channels by } 5-H D}$ significantly abolished the NF-KB-lowering influence of troxerutin $(P<0.05)$. No significant differences were found in NF-KB expression between MI/R and $\mathrm{Ml} / \mathrm{R}+5-\mathrm{HD}$ groups (Fig. 4).

\subsection{The levels of TNF- $a$}


As shown in Fig. 5, the levels of TNF-a in MI/R group was significantly increased as compared to Control group $(P<0.001)$. Preadministration of troxerutin significantly reduced the levels of TNF-a as compared to $\mathrm{MI} / \mathrm{R}$ hearts $(P<0.01)$. However, blocking the mitoK $\mathrm{ATP}_{\mathrm{P}}$ channels by 5 -HD significantly abolished the TNF-a-lowering effect of troxerutin $(P<0.05)$. No significant differences were found in TNF-a levels between Ml/R and Ml/R+ 5-HD groups (Fig. 5).

\subsection{The levels of IL-1 $\beta$}

Figure 6 shows the effect of troxerutin on the levels of IL-1 $\beta$ in treated and untreated I/R hearts. The levels of IL-1 $\beta$ in MI/R group was significantly increased as compared to Control group $(P<0.001)$.

Preconditioning with troxerutin significantly reduced the levels of IL-1 $\beta$ as compared to MI/R hearts $(P<$ 0.05). In addition, the levels of IL-1 $\beta$ were significantly decreased in troxerutin-receiving group without experiencing $\mathrm{I} / \mathrm{R}$ as compared to Control group $(\mathrm{P}<0.05)$. Blocking the mitoK $\mathrm{K}_{\mathrm{ATP}}$ channels by $5-\mathrm{HD}$ significantly abolished the IL-1 $\beta$-lowering influence of troxerutin $(P<0.05)$. No significant differences were found in the levels of IL-1 $\beta$ between MI/R and MI/R + 5-HD groups (Fig. 6).

\section{Discussion}

The present study assessed the therapeutic potentials of troxerutin in mitigation of $\mathrm{Ml} / \mathrm{R}$ damage in isolated rat heart. By evaluating clinically relevant end points, this study proved that preadministration of troxerutin is able to reduce cardiac infarct size and LDH release following MI/R compared with the untreated I/R myocardium. Besides, this study documented that the positive effects of troxerutin preconditioning may be mediated in part by its effect on inflammation. In more details, troxerutin preconditioning showed potent anti-inflammatory and cardioprotective activity via decreasing TNF-a and IL-1 $\beta$ levels. Also, troxerutin was found to suppress inflammatory response by reducing the activity of TLR4/NF-KB signaling pathway. In addition, our results showed that the beneficial effect of troxerutin was reversed by using $5-\mathrm{HD}$, as a mitoK $\mathrm{ATP}_{\text {C }}$ channel blocker. So it can be said that troxorutein may exert its cardioprotective effect under $\mathrm{MI} / \mathrm{R}$ injury by the opening of mitoK $\mathrm{ATP}_{\mathrm{AT}}$ channels.

In previous experimental studies of MI/R models, the beneficial properties of troxerutin have been evaluated. However, in order to achieve clinical goals in future, we need to widely study the effects of troxerutin on cellular signalings. For that reason, several experimental studies have assessed cardioprotective effects of troxerutin by evaluation of important cellular signalings, but the mechanism of troxerutin on inflammation via TLR4/NF-KB signaling pathway in MI/R damage has never been studied in depth. It seems that specific modulation of TLR4/NF-KB signaling pathway by troxerutin might be a novel strategy to increase survival under Ml/R injury in the future. It should be noted that the results obtained from the present study can lead to the better understanding of cardioprotective activity of troxerutin as a natural bioflavonoid in MI/R injury. The most significant observation of the current work is that troxerutin preconditioning reduced I/R injury, which was confirmed by reduction of cardiac infarct size and LDH levels. It means that pretreatment with troxerutin can increase cardiac resistance to $I / R$ injury. Our work demonstrated that cardioprotective effects of troxerutin in I/R injury were accompanied by its beneficial 
effects on inflammation through reduction of inflammatory cytokines such as TNF-a and IL-1 $\beta$ levels, as well as reduction of TLR4 and NF-KB expressions.

The specific pathophysiological mechanisms underlying MI/R injury are complex and needs further investigations; however, several studies demonstrated that inflammatory response takes part in $\mathrm{MI} / \mathrm{R}$ injury. It has been proven that an important protein which is involved in the inflammatory response of MI/R damage is TLR4 [10]. In particular, TLR4 triggers the cytokines releasing, neutrophil activation, and elevation in ROS following MI/R injury [22-24]. TLR4 plays its role via MyD88 pathway, and NF-KB can be activated through this dependant pathway, so the associated inflammatory cytokines will be released. It has been proven that the NF-KB pathway can be activated under myocardial ischemia, and can be further activated under reperfusion. So it has a decisive role in MI/R injury and participates in the stress reaction and tissue damage $[10,25]$. After translocation of NF-KB to the nucleus within the first few minutes following reperfusion, NF-KB promotes cell dysfunction and death by induction of pro-inflammatory and pro-apoptotic genes expression [4]. Therefore, inhibition of TLR4/NF-KB signaling pathway can be considered as an important therapeutic target for attenuation of MI/R injury. In consistent with previous studies, our data showed that I/R led to the increase in TLR4 and NF-KB expressions, and this accelerated inflammatory response causes a decrease in cardiac resistance to reperfusion injury. Interestingly, preadministration of troxerutin for 4 weeks could alleviate myocardial I/R-induced elevation of TLR4 and NF-KB expressions, and ultimately protect the heart against I/R damage. So it can be said that troxerutin has potent anti-inflammatory property via reducing the activity of TLR4-NF-KB pathway.

TNF- $\alpha$ and IL-1 $\beta$ play the key role in MI/R injury via causing cardiac dysfunction and cardiomyocyte necrosis and apoptosis [10]. It has been demonstrated that activation of myocardial TNF-a and TNF receptor have complex role in MI/R damage and protection from it. Excessive expression of TNF-a and stimulation of cardiomyocyte TNF receptor type 1 can lead to cardiac cell death, fibrosis, hypertrophy and contractile dysfunction. Whereas, lower expression of TNF- $a$ and stimulation of cardiomyocyte TNF receptor type 2 are protective [26]. Our study revealed that I/R caused a significant increase in the myocardial levels of TNF- $a$ and IL-1 $\beta$, which was associated with the increased cardiac injury in comparison with healthy hearts. However, pretreatment with troxerutin caused decreased in the myocardial levels of TNF- $\alpha$ and IL-1 $\beta$. Thus, positive effect of troxerutin on myocardial I/R damage may be attributed to the reduction of inflammatory cytokines such as TNF- $\alpha$ and IL-1 $\beta$ via decreasing the activity of TLR4-NF-KB signaling pathway.

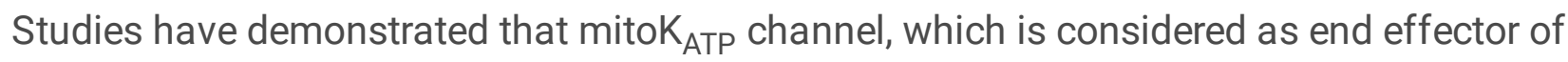
cardioprotective pathways, has cardioprotective activity in $M I / R$ injury by regulation of the mitochondrial matrix volume, and decreasing in both calcium uptake by mitochondria and mitochondrial membrane potential [27]. It has been suggested that the mito $_{\text {ATP }}$ channel opening decreases pro-apoptotic mediators and improves mitochondrial function [15], while administration of 5-HD, as a mitoK ATP $_{\text {channel }}$ blocker, reverses the reducing effect of preconditioning and postconditioning on infarct size [28]. In the present study, we showed that the anti-inflammatory effects of troxerutin were reversed when the hearts were perfused with a K-H solution containing 5-HD, 20 min before the ischemia. So it can be said that the 
positive effects of troxerutin may partly be mediated through the mitoK $\mathrm{ATP}_{\mathrm{P}}$ channels opening, and probably there is an association between the activation of mitoK $\mathrm{ATP}_{\text {chnels }}$ chand inhibitory effect of troxerutin on inflammatory mediators under MI/R injury, but needs to be investigated in depth. In addition, it seems that the activation of mitoK $\mathrm{K}_{\text {ATP }}$ channels by troxerutin can protect the functional integrity of mitochondria, prevent the mitochondria swelling, and inhibit the mitochondrial permeability transition pore (mPTP) opening, leading to the decreased production and releasing of inflammatory mediators from the mitochondria in $\mathrm{MI} / \mathrm{R}$ condition.

Conforming to our study, many previous researches demonstrated that troxerutin has protective effects against cardiovascular diseases, and oxidative and inflammatory damages in numerous organs [16-19, $29,30]$. Troxerutin has been shown to inhibit the endoplasmic reticulum stress pathway and reduce intracellular inflammatory proteins and enzyme activities including COX2, JNK1/IKKb, iNOS, and NF-KB in specific tissues of healthy rats [20,30-32]. Moreover, in our previous works, we have indicated that troxerutin preconditioning could protect the diabetic I/R hearts by prevention of myocardial apoptosis through glycogen synthase kinase-3 $\beta$ (GSK-3 $\beta$ ) phosphorylation [33]. In addition, we proved that troxerutin preconditioning had protective effects in doxorubicin-induced cardiotoxicity in rats via antioxidative activities and restoration of mitochondrial function and the expression profiles of cardiac SIRT1/PGC-1a/NRF-2 network [34].

\section{Conclusions}

The results of the present work contribute to some valuable conclusions. Preconditioning with troxerutin could provide cardioprotective effects under $\mathrm{Ml} / \mathrm{R}$ damage which were proven by reduction of myocardial infarct size and LDH release. Cardioprotective effects of troxerutin were partly mediated through modulation of inflammatory response including reduction of TNF- $\alpha$ and IL-1 $\beta$ via decreasing the activity of TLR4/NF-KB signaling pathway. We also indicated that the opening of mitoK $\mathrm{ATP}_{\text {P }}$ channels play a crucial role in the cardioprotective effects of troxerutin in MI/R injury condition. However, further attempts are required to examine the effects of troxerutin on the other important pathways involved in heart protection under I/R injury.

\section{Declarations}

\section{Funding}

The author(s) disclosed receipt of the following financial support for the research, authorship, and/or publication of this article: This work has been supported by a grant (grant number: 60872) from Drug Applied Research Center, and Immunology Research Center, Tabriz University of Medical Sciences, Tabriz, Islamic Republic of Iran.

\section{Conflicts of interest}


The author(s) declared no potential conflicts of interest with respect to the research, authorship, and/or publication of this article.

\section{Availability of data and material}

The authors confirm that the data supporting the findings of this study are available within the article [and/or] its supplementary materials.

\section{Code availability}

Not applicable.

\section{Authors' contributions}

FM and BM performed the experimental tests, gathered and analyzed the data, and drafted the manuscript. SG and AJ performed the experimental tests. RB did the study design, supervised the whole project, contributed in interpretation of the results and finalized the manuscript editing and critically revised the manuscript. All gave final approval and agree to be accountable for all aspects of work ensuring integrity and accuracy.

\section{Ethics approval}

Animal care procedures, as well as all experimental protocols were approved by the Institutional Animal Ethical Committee of Tabriz University of Medical Sciences (Ethical code: IR.TBZMED.VCR.REC.92-3729).

\section{Acknowledgment}

None.

\section{References}

1. Ingelsson E et al (2006) Socioeconomic factors as predictors of incident heart failure. J Card Fail 12(7):540-545

2. Badalzadeh R, Yavari R, Chalabiani D (2015) Mitochondrial ATP-sensitive $K$ + channels mediate the antioxidative influence of diosgenin on myocardial reperfusion injury in rat hearts. Gen Physiol Biophys 34(3):323-329

3. Badalzadeh R et al (2017) Chronic type-I diabetes could not impede the anti-inflammatory and antiapoptotic effects of combined postconditioning with ischemia and cyclosporine $A$ in myocardial reperfusion injury. Journal of physiology biochemistry 73(1):111-120

4. Arslan F et al., TLR2 and TLR4 in ischemia reperfusion injury. Mediators of inflammation, 2010. 2010

5. Ferdinandy P, Schulz R, Baxter GF (2007) Interaction of cardiovascular risk factors with myocardial ischemia/reperfusion injury, preconditioning, and postconditioning. Pharmacol Rev 59(4):418-458 
6. Moens AL et al (2005) Myocardial ischemia/reperfusion-injury, a clinical view on a complex pathophysiological process. Int J Cardiol 100(2):179-190

7. Yellon DM, Hausenloy DJ (2007) Myocardial reperfusion injury. N Engl J Med 357(11):1121-1135

8. Bayrami G et al (2018) Effect of ischemic postconditioning on myocardial function and infarct size following reperfusion injury in diabetic rats pretreated with vildagliptin. J Cardiovasc Pharmacol Therap 23(2):174-183

9. Latini R, Brines M, Fiordaliso F (2008) Do non-hemopoietic effects of erythropoietin play a beneficial role in heart failure? Heart Fail Rev 13(4):415-423

10. Chen $\mathrm{H}$ et al (2016) Mechanism of TLR-4/NF-KB pathway in myocardial ischemia reperfusion injury of mouse. Asian Pacific journal of tropical medicine 9(5):503-507

11. Saini HK et al (2005) Role of tumour necrosis factor-alpha and other cytokines in ischemiareperfusion-induced injury in the heart. Experimental Clinical Cardiology 10(4):213

12. Frangogiannis NG, Interleukin-1 in cardiac injury, repair, and remodeling: pathophysiologic and translational concepts. Discoveries, 2015. 3(1)

13. Miura T et al (2001) Mitochondrial ATP-sensitive $\mathrm{K}+$ channels play a role in cardioprotection by Na+$\mathrm{H}+$ exchange inhibition against ischemia/reperfusion injury. J Am Coll Cardiol 37(3):957-963

14. Zweier JL (1988) Measurement of superoxide-derived free radicals in the reperfused heart. Evidence for a free radical mechanism of reperfusion injury. J Biol Chem 263(3):1353-1357

15. Feyzizadeh $S$ et al (2018) Signaling mediators modulated by cardioprotective interventions in healthy and diabetic myocardium with ischaemia-reperfusion injury. European journal of preventive cardiology 25(14):1463-1481

16. Fan SH et al (2009) Troxerutin protects the mouse kidney from d-galactose-caused injury through anti-inflammation and anti-oxidation. Int Immunopharmacol 9(1):91-96

17. Kessler $\mathrm{M}$ et al (2002) Free radical scavenging and skin penetration of troxerutin and vitamin derivatives. J Dermatolog Treat 13(3):133-141

18. Siegers CP, Syed Ali S, Tegtmeier M (2008) Aescin and troxerutin as a successful combination for the treatment of inner ear perfusion disturbances. Phytomedicine 15(3):160-163

19. Boisseau MR et al (1995) Fibrinolysis and hemorheology in chronic venous insufficiency: a double blind study of troxerutin efficiency. J Cardiovasc Surg (Torino) 36(4):369-374

20. Liu C-M, Ma J-Q, Lou Y (2010) Chronic administration of troxerutin protects mouse kidney against dgalactose-induced oxidative DNA damage. Food Chem Toxicol 48(10):2809-2817

21. Najafi $\mathrm{M}$ et al (2018) Anti-arrhythmogenic and anti-inflammatory effects of troxerutin in ischemia/reperfusion injury of diabetic myocardium. Biomed Pharmacother 102:385-391

22. Aikawa R et al (2001) Reactive oxygen species in mechanical stress-induced cardiac hypertrophy. Biochem Biophys Res Commun 289(4):901-907

23. Hernandez-Resendiz S et al (2018) The Role of Redox Dysregulation in the Inflammatory Response to Acute Myocardial Ischaemia-reperfusion Injury - Adding Fuel to the Fire. Curr Med Chem 
25(11):1275-1293

24. Vilahur $G$, Badimon $L$ (2014) Ischemia/reperfusion activates myocardial innate immune response: the key role of the toll-like receptor. Front Physiol 5:496

25. Kis A, Yellon DM, Baxter GF (2003) Role of nuclear factor-kB activation in acute ischaemiareperfusion injury in myocardium. Br J Pharmacol 138(5):894-900

26. Kleinbongard P, Schulz R, Heusch G (2011) TNFa in myocardial ischemia/reperfusion, remodeling and heart failure. Heart Fail Rev 16(1):49-69

27. Lu L et al (2008) Thiazolidinedione drugs block cardiac KATP channels and may increase propensity for ischaemic ventricular fibrillation in pigs. Diabetologia 51(4):675-685

28. Flagg TP, Nichols CG (2005) Sarcolemmal K(ATP) channels: what do we really know? J Mol Cell Cardiol 39(1):61-70

29. Badalzadeh R et al (2017) Troxerutin Preconditioning and Ischemic Postconditioning Modulate Inflammatory Response after Myocardial Ischemia/Reperfusion Injury in Rat Model. Inflammation 40(1):136-143

30. Zhang Z-F et al (2014) Troxerutin improves hepatic lipid homeostasis by restoring NAD+-depletionmediated dysfunction of lipin 1 signaling in high-fat diet-treated mice. Biochem Pharmacol 91(1):74-86

31. Lu J et al (2013) Troxerutin counteracts domoic acid-induced memory deficits in mice by inhibiting CCAAT/enhancer binding protein beta-mediated inflammatory response and oxidative stress. $J$ Immunol 190(7):3466-3479

32. Lu J et al (2010) Chronic administration of troxerutin protects mouse brain against d-galactoseinduced impairment of cholinergic system. Neurobiol Learn Mem 93(2):157-164

33. Mokhtari B et al (2015) Phosphorylation of GSK-3 $\beta$ and reduction of apoptosis as targets of troxerutin effect on reperfusion injury of diabetic myocardium. Eur J Pharmacol 765:316-321

34. Babaei-Kouchaki S et al (2020) Effect of troxerutin on oxidative stress and expression of genes regulating mitochondrial biogenesis in doxorubicin-induced myocardial injury in rats. Naunyn Schmiedebergs Arch Pharmacol 393(7):1187-1195

\section{Figures}




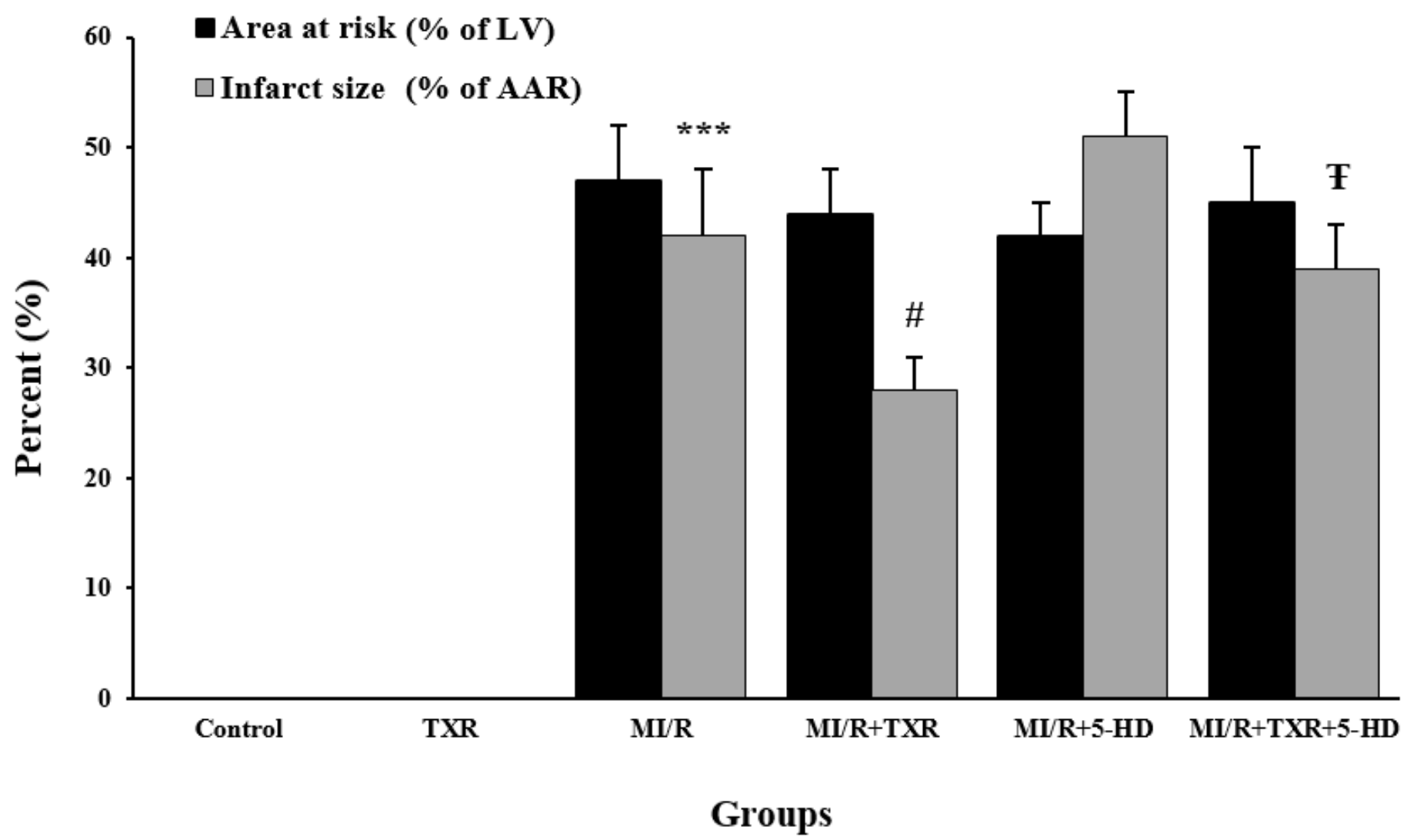

Figure 1

Area at risk (AAR) and infarct size (IS) percentages in different groups. The data were expressed as mean \pm SEM ( $n=5$ for each group). ( ${ }^{\star * \star} P<0.001$ vs. Control group, $\# \mathrm{P}<0.05$ vs. Ml/R group, and $\mp \mathrm{P}<0.05$ vs. MI/R + TXR group). TXR: troxerutin; MI/R: myocardial ischemia/reperfusion; MI/R + TXR: myocardial ischemia/reperfusion + troxerutin; Ml/R + 5-HD: myocardial ischemia/reperfusion + 5-hydroxydecanoate; $\mathrm{Ml} / \mathrm{R}+\mathrm{TXR}+$ 5-HD: myocardial ischemia/reperfusion + troxerutin + 5-hydroxydecanoate. 


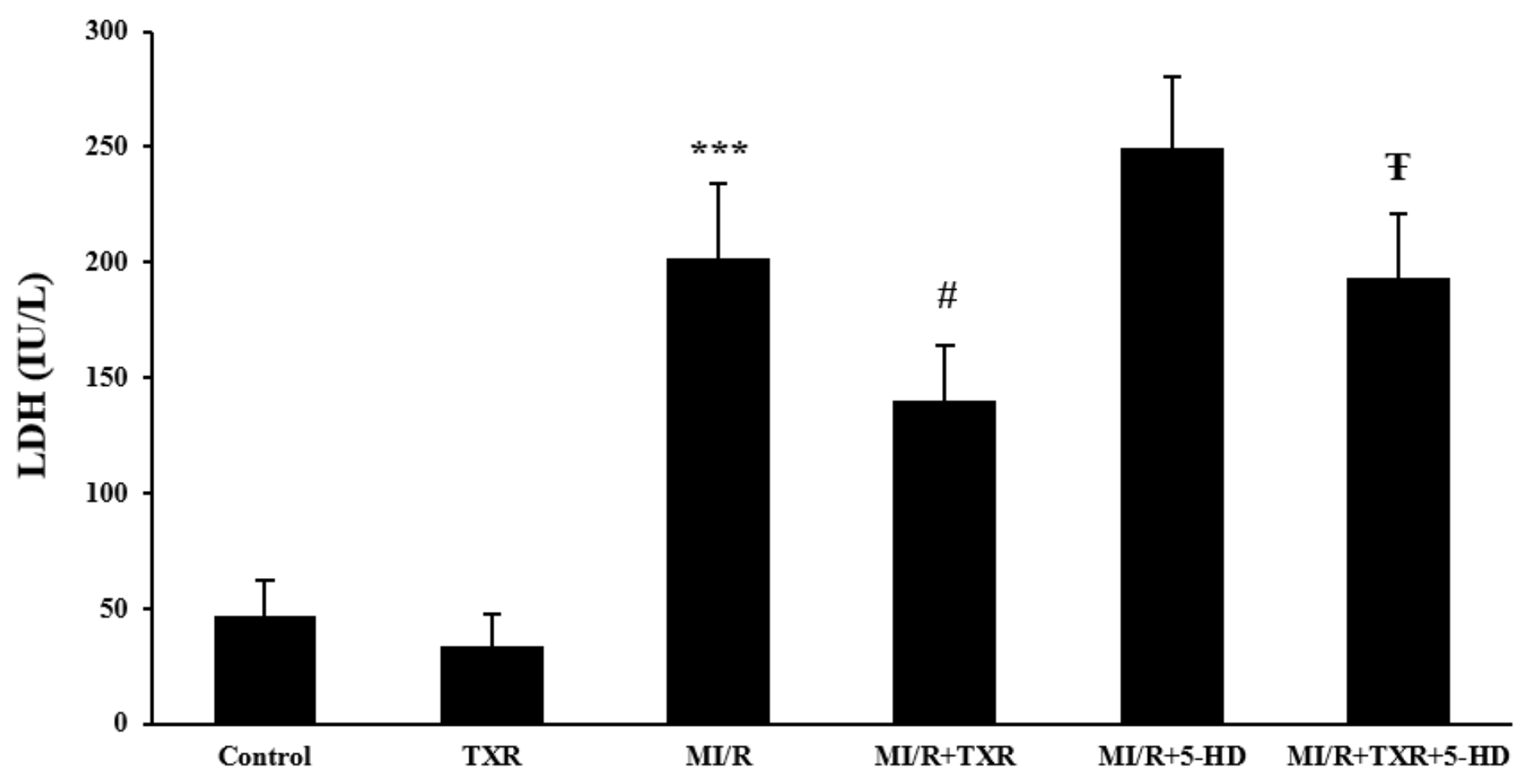

\section{Groups}

Figure 2

Lactate dehydrogenase (LDH) release into the coronary effluent in different groups. The data were expressed as mean \pm SEM ( $n=5$ for each group). ( ${ }^{* \star \star} P<0.001$ vs. Control group, $\# P<0.05$ vs. MI/R group, and $\mp \mathrm{P}<0.05 \mathrm{vs}$. Ml/R + TXR group). TXR: troxerutin; Ml/R: myocardial ischemia/reperfusion; $\mathrm{MI} / \mathrm{R}+$ TXR: myocardial ischemia/reperfusion + troxerutin; MI/R + 5-HD: myocardial ischemia/reperfusion + 5-hydroxydecanoate; $\mathrm{MI} / \mathrm{R}+\mathrm{TXR}+5-\mathrm{HD}$ : myocardial ischemia/reperfusion + troxerutin + 5-hydroxydecanoate. 


\section{TLR4}

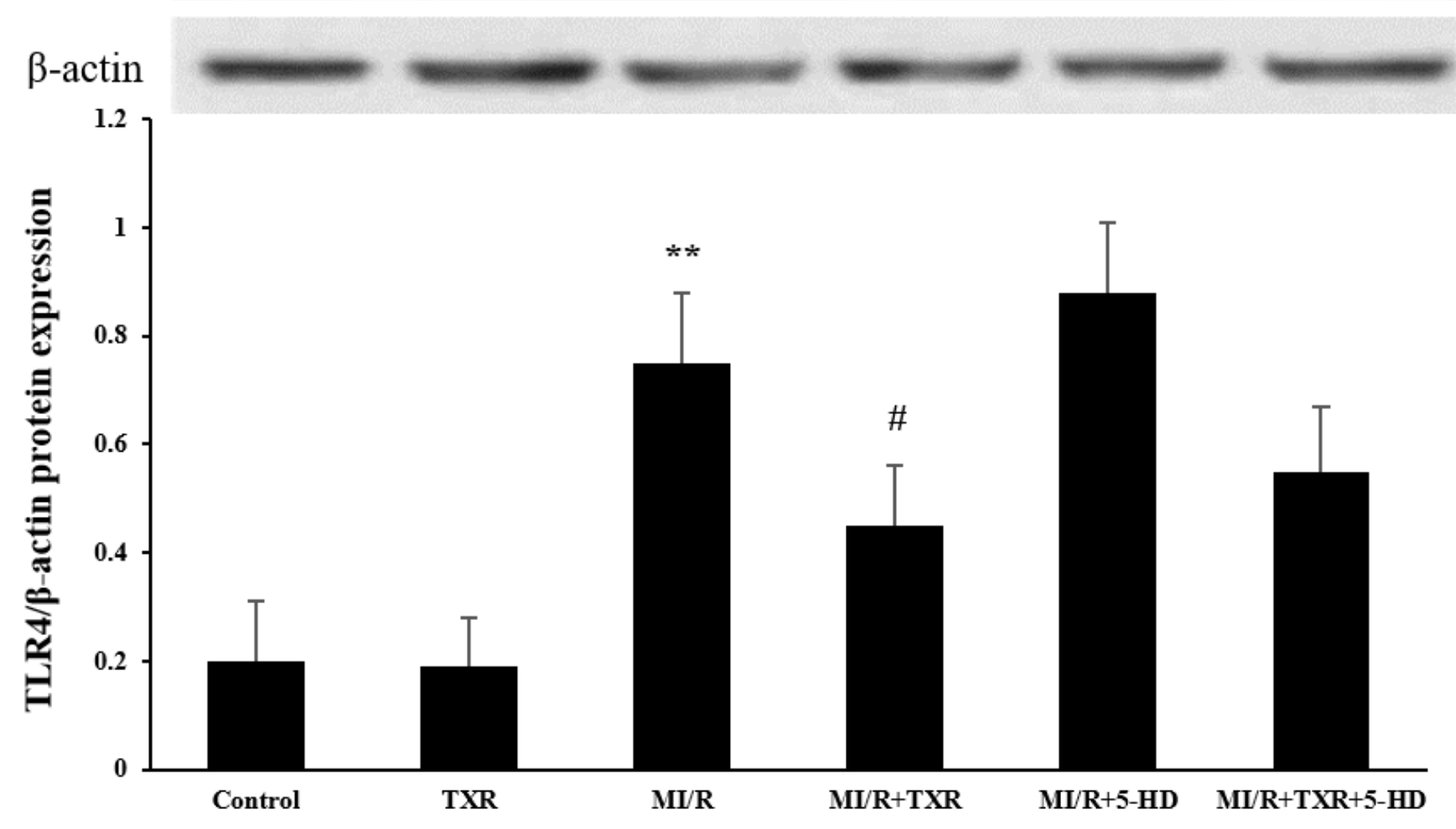

Groups

\section{Figure 3}

Western blot band analysis and densitometry of TLR4 correlated to the $\beta$-actin band in different groups. The data were expressed as mean \pm SEM ( $n=5$ for each group). (** $P<0.01$ vs. Control group, and \# $P<$ 0.05 vs. MI/R group). TXR: troxerutin; MI/R: myocardial ischemia/reperfusion; MI/R + TXR: myocardial ischemia/reperfusion + troxerutin; MI/R + 5-HD: myocardial ischemia/reperfusion + 5-hydroxydecanoate; $\mathrm{Ml} / \mathrm{R}+\mathrm{TXR}+5-\mathrm{HD}$ : myocardial ischemia/reperfusion + troxerutin + 5-hydroxydecanoate. 


\section{$\mathrm{NF}-\mathrm{\kappa B}$}

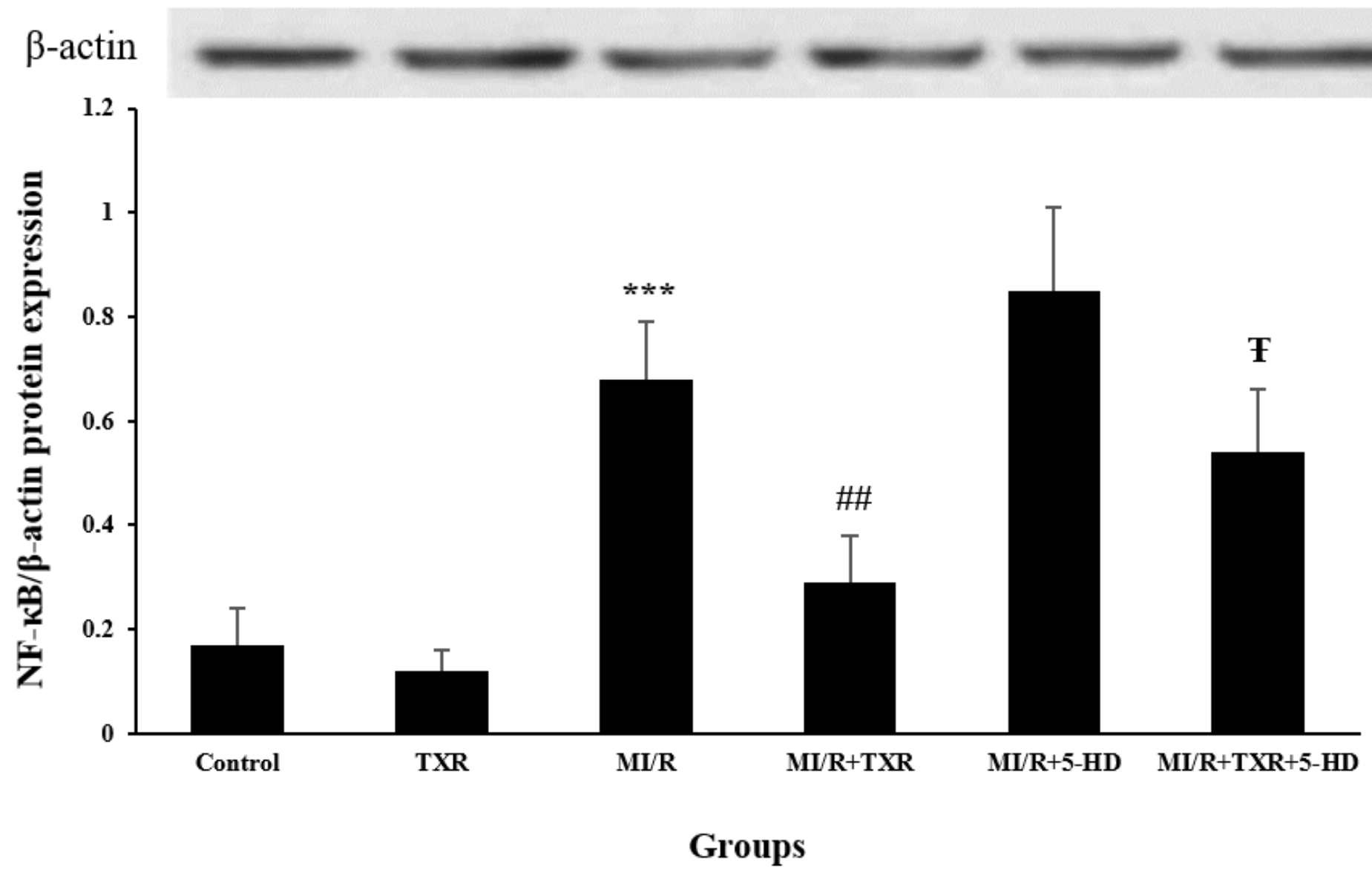

\section{Figure 4}

Western blot band analysis and densitometry of NF-KB correlated to the $\beta$-actin band in different groups. The data were expressed as mean \pm SEM ( $n=5$ for each group). ( ${ }^{\star \star \star} P<0.001$ vs. Control group, \#\# $P<$ 0.01 vs. Ml/R group, and $\mp \mathrm{P}<0.05$ vs. Ml/R + TXR group). TXR: troxerutin; Ml/R: myocardial ischemia/reperfusion; MI/R + TXR: myocardial ischemia/reperfusion + troxerutin; MI/R + 5-HD: myocardial ischemia/reperfusion + 5-hydroxydecanoate; MI/R + TXR + 5-HD: myocardial ischemia/reperfusion + troxerutin +5 -hydroxydecanoate. 


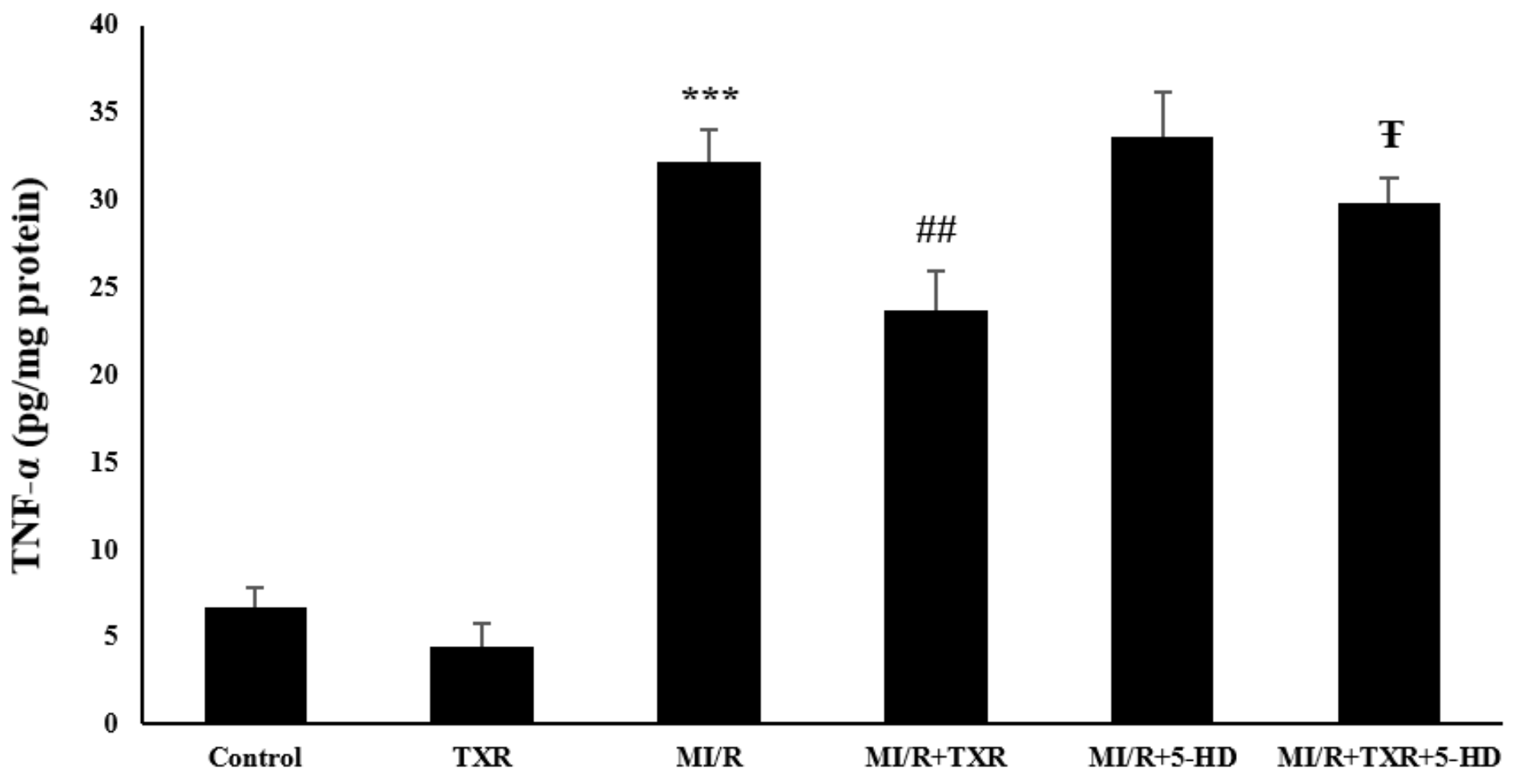

\section{Groups}

Figure 5

The levels of TNF- $a$ in different groups. The data were expressed as mean \pm SEM ( $n=5$ for each group).

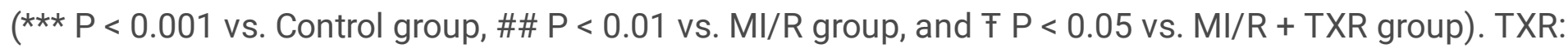
troxerutin; MI/R: myocardial ischemia/reperfusion; MI/R + TXR: myocardial ischemia/reperfusion + troxerutin; MI/R + 5-HD: myocardial ischemia/reperfusion + 5-hydroxydecanoate; MI/R + TXR + 5-HD: myocardial ischemia/reperfusion + troxerutin + 5-hydroxydecanoate. 


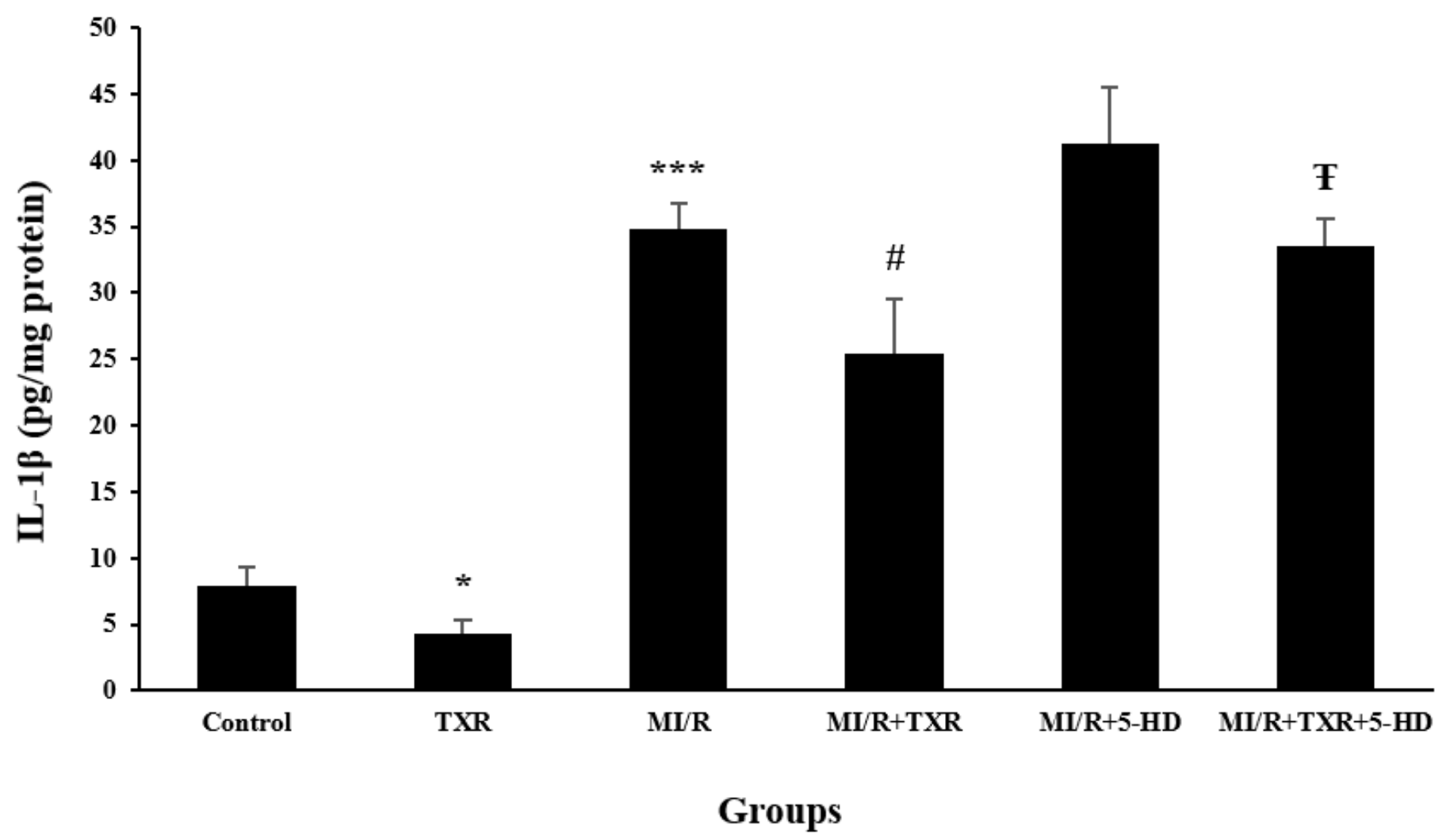

Figure 6

The levels of IL-1 $\beta$ in different groups. The data were expressed as mean \pm SEM ( $n=5$ for each group). (* $P<0.05$ and $* \star \star P<0.001$ vs. Control group, \# $P<0.05$ vs. Ml/R group, and $\mp P<0.05$ vs. Ml/R $+T X R$ group). TXR: troxerutin; MI/R: myocardial ischemia/reperfusion; MI/R + TXR: myocardial ischemia/reperfusion + troxerutin; Ml/R + 5-HD: myocardial ischemia/reperfusion + 5-hydroxydecanoate; $\mathrm{Ml} / \mathrm{R}+\mathrm{TXR}+$ 5-HD: myocardial ischemia/reperfusion + troxerutin + 5-hydroxydecanoate. 